\title{
Early recognition of intraventricular hemorrhage in the setting of thrombocytosis in the emergency department
}

This article was published in the following Dove Press journal:

Open Access Emergency Medicine

12 May 2016

Number of times this article has been viewed

\section{Marvinia Charles \\ Romy Fontoura \\ Gregory Sugalski}

Department of Emergency Medicine, Rutgers New Jersey Medical School, Newark, NJ, USA
Correspondence: Marvinia Charles Department of Emergency Medicine, Rutgers New Jersey Medical School, I50 Bergen Street, Newark, NJ 07I0I, USA

Tel + I 9739729261

Fax + I 9739726646

Email rmf82@njms.rutgers.edu

\begin{abstract}
Thrombocytosis is frequently encountered as an incidental laboratory finding since isolated thrombocytosis is often asymptomatic. Even though thrombocytosis is benign and selflimiting in most cases, it can at times result in thrombosis or hemorrhage. The most common type of thrombocytosis is reactive (secondary) thrombocytosis and can be due to infections, trauma, surgery, or occult malignancy. Since thrombocytosis is a known risk factor for thrombosis, it is commonly a concern for ischemic stroke and myocardial infarction. Much less common are hemorrhagic events associated with thrombocytosis. Studies have shown that when hemorrhage is present in patients with thrombocytosis, it is most often seen in the setting of chronic myelogenous leukemia and essential thrombocythemia. In essential thrombocythemia, the overall risk of bleeding and thrombosis is $0.33 \%$ per patient-year and $6.6 \%$ per patient-year, respectively. In the general population, the risk of bleeding and thrombosis is $0 \%$ and $1.2 \%$, respectively. The present study is a case report of an 83-year-old man who presented to the emergency department with hypertension and headache, who was then found to have significant thrombocytosis (platelets $>1,000 \times 10^{9} / \mathrm{L}$ ) and acute right intraventricular hemorrhage without any signs of neurological deficits, or evidence of vascular malformations or mass. We present this case report for review and discussion of some of the challenges and considerations associated with the management of such patients.
\end{abstract}

Keywords: thrombocytosis, thrombocythemia, hemorrhage, intraventricular hemorrhage, thrombosis, headache, emergency department

\section{Introduction}

Thrombocytosis is commonly defined by a platelet count $>450,000 / \mathrm{mm}^{3}$ (or $\mu \mathrm{L}$ ) $\left(150-450 \times 10^{9} / \mathrm{L}\right)$. Extreme thrombocytosis is an even higher platelet count, one that exceeds $1,000 \times 10^{9} / \mathrm{L}$. The etiology of a high platelet count can be a reactive (secondary) cytokine-driven mechanism or may be the result of growth factor-independent overproduction of platelets by clonal megakaryocytes, as in the case of myeloproliferative disorders (MPDs) or myelodysplastic disorders (autonomous thrombocytosis). ${ }^{1}$ Reactive thrombocytosis is the most common cause of thrombocytosis and is more common in association with infection, patients with surgical disease, iron-deficiency anemia, and malignancy. ${ }^{2}$ Approximately $10 \%$ of all reported patients with platelet counts $>1$ million have thrombocytosis secondary to rheumatoid and collagen vascular diseases, such as rheumatoid arthritis. ${ }^{3}$ In patients postsplenectomy, reactive thrombocytosis has an incidence of $\sim 75 \%-82 \%$, and platelet counts can remain elevated for several weeks. ${ }^{4}$ Reactive thrombocytosis is usually, but not always, characterized by an elevated C-reactive protein or erythrocyte sedimentation rate, and platelets are usually small with a normal mean platelet volume. ${ }^{5}$ 
Autonomous thrombocytosis is defined as a thrombocytosis in the presence of an established diagnosis of chronic MPDs. Essential thrombocythemia (ET) is one of the four subgroups of chronic MPDs. The classical MPDs include ET, polycythemia vera, and primary myelofibrosis, as well as chronic myeloid leukemia (one of the MPDs that exhibits a specific cytogenetic abnormality). The diagnosis of ET is one of exclusion: once the other chronic MPDs and thrombocytosis from reactive (secondary) causes have been excluded, the diagnosis of ET can be made. The World Health Organization's criterion for the diagnosis of ET is broader and includes: 1) a persistently elevated platelet count $>450,000$ / $\mu \mathrm{L}$ in the absence of reactive thrombocytosis; 2) normal iron stores, as evidenced by normal serum ferritin and normal red cell mean corpuscular volume. It requires the failure of iron replacement therapy to increase hemoglobin levels to the polycythemia vera range in the presence of decreased serum ferritin; 3) megakaryocytic hyperplasia on bone marrow aspiration and biopsy; and 4) the absence of Philadelphia chromosome or the $B C R / A B L$ gene rearrangement, as well as the absence of peripheral blood, bone marrow, and karyotypic evidence for a myelodysplastic disorder.

While the presence of JAK2 mutation is highly specific for the diagnosis of an MPD (eg, polycythemia vera, ET, and primary myelofibrosis), it is present only in 50-64\% of patients with ET and is absent in patients with reactive thrombocytosis. A calreticulin mutation is found in $15-25 \%$ of patients with ET, and an MPL proto-oncogene, thrombopoietin receptor mutation is found in an additional $4 \% .{ }^{6} \mathrm{ET}$ can be familial or sporadic. In the familial ET, unlike in sporadic ET, activating mutations in the genes for thrombopoietin or c-Mpl, or mutations in the genes for other proteins are responsible for thrombopoietin-mediated thrombocytosis, and is inherited in an autosomal dominant pattern. ${ }^{6}$

Regardless of the etiology, a high platelet count has the potential to be associated with vasomotor (headache, visual symptoms, lightheadedness, and atypical chest pain), thrombotic, or hemorrhagic complications. These complications are much more likely to occur in association with autonomous thrombocytosis and not with reactive thrombocytosis. ${ }^{7}$

\section{Case}

This is a case of an 83-year-old man who presented to the emergency department with a right-sided headache for 2 days. The headache was described as a pain level of 8/10, throbbing, and radiating to the back of the neck. No weakness, numbness, visual deficits, nausea, vomiting, dizziness, or lightheadedness was reported. He denied any difficulty in chewing or swallowing and denied any falls or trauma to the head. He has a history of hypertension, type 2 diabetes mellitus, osteoarthritis, and glaucoma.

His physical examination was significant for a nonfocal neurological examination, chronic poor visual acuity, and weak right palmar grasp secondary to his arthritis.

In the emergency department, his vital signs were as follows: height, $172.7 \mathrm{~cm}$; weight, $72.6 \mathrm{~kg}$; blood pressure, $188 / 109 \mathrm{mmHg}$; heart rate, $60 \mathrm{bpm}$; temperature, $36.8^{\circ} \mathrm{C}$; respiratory rate, $18 \mathrm{bpm}$. The following laboratory data were remarkable: white blood cells, $8.810^{9}$ cells/L; red blood cells, $3.04 \mathrm{M} / \mu \mathrm{L}$; hematocrit, 30.3\%; hemoglobin, $10.9 \mathrm{gm} / \mathrm{dl}$; platelets $1,097 \times 10^{9} / \mathrm{L}$; mean corpuscular volume, $99.8 \mathrm{fL}$; basic metabolic panel was notable for creatinine of $1.7 \mathrm{mg} / \mathrm{dL}$ and a normal glucose of $5.73 \mathrm{mmol} / \mathrm{L}$. Coagulation studies were as follows: prothrombin time, $15.7 \mathrm{~s}$; partial thromboplastin time, $33.7 \mathrm{~s}$; international normalized ratio, 1.2.

His medications were famotidine $20 \mathrm{mg}$ daily, amlodipine $10 \mathrm{mg}$ daily, ultrashort-acting insulin 2-6 U subcutaneous four times a day, nicardipine, and lisinopril $20 \mathrm{mg}$ daily. Initially, the management was focused on conservative blood pressure control and antiplatelet therapy with aspirin. A subsequent head computerized tomography (CT) revealed a right intraventricular hemorrhage (Figure 1), which was managed with platelet transfusions, desmopressin acetate (DDAVP), and a nicardipine drip. The patient was admitted to the medical intensive care unit for frequent neurological examination and continuous blood pressure monitoring and control. The patient had an 11-day hospital stay and an extensive negative workup. Cardiac echocardiogram, vascular duplex of the lower extremities, and carotid duplex

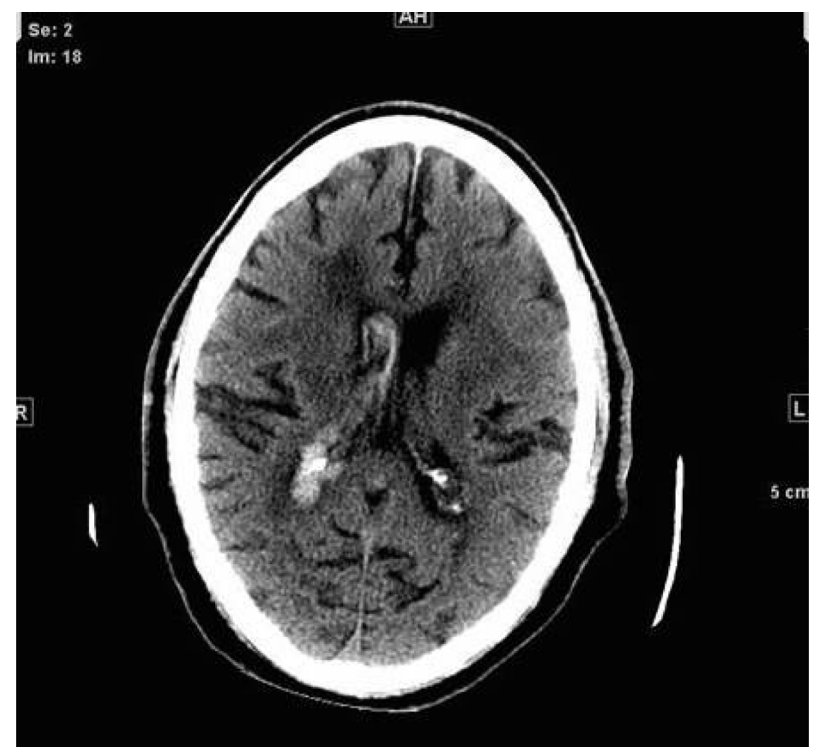

Figure I Noncontrast brain CT: acute intraventricular hemorrhage in the right lateral ventricle with small hemorrhage in the atrium of the left ventricle. Abbreviations: $\mathrm{AH}$, anterior head; $\mathrm{CT}$, computerized tomography; L, left; $\mathrm{R}$, right. 


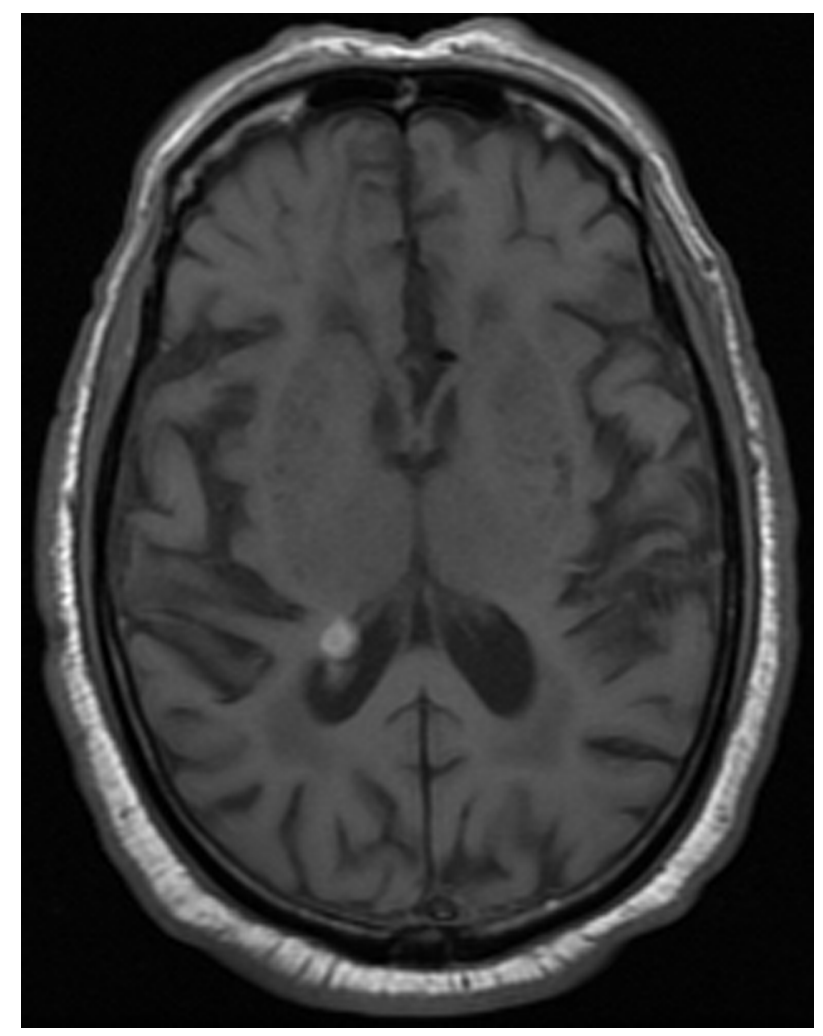

Figure 2 Noncontrast TI-weighted brain MRI: image demonstrates hemorrhage originating from ependyma of right lateral ventricle.

Abbreviation: MRI, magnetic resonance imaging.

were negative. Follow-up imaging showed stable intraventricular hemorrhage (IVH) (Figure 2), and the patient was ultimately discharged to Kessler Rehabilitation Center with zero neurological deficits.

\section{Discussion}

\section{Thrombocytosis and presumptive diagnosis of ET}

It is important to note that the purpose of this case study is to recognize the hemorrhagic complications of thrombocytosis and the management of IVH in this setting. Secondary causes of thrombocytosis were ruled out. This patient's total iron was low at $28 \mu \mathrm{g} / \mathrm{dL}$ (normal: $59-158 \mu \mathrm{g} / \mathrm{dL}$ ), and his total iron binding capacity was also low at $190 \mu \mathrm{g} / \mathrm{dL}$, thus, ruling out iron-deficiency anemia as a cause for his thrombocytosis. In addition, there was no known trauma or recent surgery, no functional asplenia, or signs of hemolysis. Therefore, there was little evidence to support reactive thrombocytosis. In the absence of other known chronic MPDs, bone marrow biopsy and JAK2 mutation screening were suggested, and a presumptive diagnosis of ET was made. JAK2617V $>$ F, a Janus kinase 2 mutation, is myeloid neoplasm specific; its presence excludes secondary polycythemia, thrombocytosis, or bone marrow fibrosis from other causes. More than $50 \%$ of patients with ET exhibit a $J A K 2617 \mathrm{~V}>$ F mutation, and thus, $J A K 2$ mutation screening can be complementary to histology for the diagnosis of ET. ${ }^{6}$ This patient was lost to follow-up, and a quantitative and/or qualitative $J A K 2617 \mathrm{~V}>\mathrm{F}$ mutation screening was not performed.

\section{The intraventricular hemorrhage}

Using the database of published works cited in the Index Medicus (www.pubmed.com), the operative definition of extreme thrombocytosis is a platelet count that exceeds $1,000 \times 10^{9} / \mathrm{L}$. In extreme thrombocytosis and ET, hemorrhage is 20 times less frequent than in thrombotic events. ${ }^{1}$ When bleeding does occur in association with thrombocytosis, it is most often mucocutaneous, such as echymosis, epistaxis, menorrhagia, and gingival bleeding. The most severe case of bleeding is intraventricular/intracerebral bleeding. ${ }^{8}$

Once platelet levels rise $>1-1.5$ million $/ \mathrm{mL}$, patients are at increased risk for hemorrhagic events. The major risk factor for hemorrhage in ET appears to be extreme thrombocytosis. In this setting, the acquisition of von Willebrand factor (vWF) deficiency due to the loss of intermediate and large $\mathrm{vWF}$ multimers is a proposed rationale for the paradoxical bleeding. The arterial thrombotic condition in thrombocythemia changes into an overt spontaneous bleeding tendency at mean platelet counts of $\sim 2,000 \pm 1,000 \times 10(9) / \mathrm{L}$ due to an acquired von Willebrand disease with normal values of vWF antigen concentration but absence of intermediate and large vWF multimers in plasma. ${ }^{9}$ The diagnosis of acquired von Willebrand syndrome with MPDs is difficult, and most cases have been diagnosed by chance since bleeding symptoms are mild..$^{10}$ In general, the laboratory features of acquired von Willebrand syndrome in MPDs are characterized by an increased platelet count of unknown etiology, normal partial thromboplastin time, slightly increased bleeding time, and slightly reduced factor VIII:C activity. ${ }^{10}$ It follows that it is possible that our patient may have acquired a von Willebrand defect secondary to the extreme thrombocytosis and possible ET. Yet, in the absence of a value for bleeding time, vWF antigen and factor VIII:C levels, ristocetin, and collagenbinding assays, we are unable to determine if indeed this patient had acquired von Willebrand defect.

\section{Management}

Patient management guidelines for these complex patients are nebulous at best; antithrombotic therapy, active platelet transfusions, coagulopathy correction, tight blood pressure control, and/or cytoreductive therapy are just a few of the decisions that must be made in a rapid, evidence-based rubric. Half of the patients with ET are completely asymptomatic, 
and the remaining half present with vague vasomotor symptoms. Yet, bleeding manifestations at initial presentation of ET are relatively frequent; the risk is significantly associated with extreme thrombocytosis. ${ }^{11}$

In this case, the patient was first started on antiplatelet aggregation therapy with aspirin being administered for vague complaints of chest pain and hypertension in the setting of severe thrombocytosis. A head CT was obtained and revealed a right-sided intraventricular hemorrhage. As expected, platelet function tests came back low at 180 PRU (194-418 PRU). Aspirin therapy was discontinued, and the patient was then immediately transfused a six-pack of platelets and administered DDAVP (glomerular filtration rate $=40 \mathrm{~mL} / \mathrm{min}$, creatinine $=1.7 \mathrm{mg} / \mathrm{dL}$ ). DDAVP exerts a substantial hemostatic effect by inducing the release of vWF from its storage sites in endothelial cells. It has proved useful in treating (or preventing) bleeding episodes in patients with platelet function defects. ${ }^{12}$ In addition, the patient received a Nicardipine drip for tight hemodynamic control with a goal of systolic blood pressure $<140 \mathrm{mmHg}$ and the patient was then transferred to the medical intensive care unit for observation and monitoring with frequent neurological examination. Repeat head CT showed stable intraventricular hemorrhage, and the patient was weaned off the Cardene drip and transitioned back to his home antihypertensive medications. Follow-up brain magnetic resonance imaging was consistent with a stable IVH and showed signs of remote intraparenchymal hemorrhage with encephalomalacia and hemosiderin deposition. In addition, there were multiple scattered punctate foci throughout the brain parenchyma and brainstem due to remote microhemorrhages concerning for old neurological insults possibly from prolonged untreated thrombocytosis in this patient with hypertension.

Within 11 days, this patient was discharged to Kessler Rehabilitation Center with zero neurological deficits. The patient never followed up as scheduled with hematology. As such, definitive diagnosis and appropriate treatment with lifestyle modifications (such as smoking cessation), platelet-lowering agents, and possible thrombocytophoresis were not possible.

Early recognition and cautious medical management in this patient clearly seems to have so far minimized this patient's sequelae. The case illustrates a little appreciated but highly important complication of extreme thrombocytosis. Certainly, every headache does not require a head CT.
However, practitioners must recognize all the possible complications of thrombocytosis in order to safely evaluate patients presenting in this manner. In this case, we believe that the early head CT in this elderly patient with thrombocytosis played a key role in guiding us away from antiplatelet therapy and directly contributed to his favorable final outcome.

\section{Acknowledgment}

Institutional review board ethics approval for this case report was not required by the institutional review board of Rutgers New Jersey Medical School, as the activities described in the approval application did not meet the regulatory definition of human subjects research provided in 45 CFR 46.102. The authors advise they were unable to obtain the patient's consent for this report as the activities described in the approval application did not meet the regulatory definition of human subjects research provided in 45 CFR 46.102.

\section{Disclosure}

The authors report no conflicts of interest in this work.

\section{References}

1. Schafer AI. Thrombocytosis. N Engl J Med. 2004;350(12): 1211-1219.

2. Santhosh-Kumar CR, Yohannan MD, Higgy KE, al-Mashhadani SA. Thrombocytosis in adults: analysis of 777 patients. J Intern Med. 1991;229(6):493-495.

3. Farr MM. Thrombocytosis of active rheumatoid disease. Ann Rheum Dis. 1983;42(5):545-549.

4. Khan PN, Nair RJ, Olivares J, Tingle LE, Li Z. Postsplenectomy reactive thrombocytosis. Proc (Bayl Univ Med Cent). 2009;22(1):9-12.

5. Harrison $\mathrm{CN}$. Guideline for investigation and management of adults and children presenting with a thrombocytosis. Br J Haematol. 2010;149(3):352-375.

6. Tefferi A, Thiele J, Orazi A, et al. Proposals and rationale for revision of the World Health Organization diagnostic criteria for polycythemia vera, essential thrombocythemia, and primary myelofibrosis: recommendations from an ad hoc international expert panel. Blood. 2007; 110(4):1092-1097.

7. Randi ML, Stocco F, Rossi C, Tison T, Girolami A. Thrombosis and hemorrhage in thrombocytosis: evaluation of a large cohort of patients (357 cases). J Med. 1991;22(4-5):213-223.

8. Elliott MA, Elliott MA, Tefferi A. Thrombosis and haemorrhage in polycythaemia vera and essential thrombocythaemia. Br J Haematol. 2005;128(3):275-290.

9. Michiels JJ. Acquired von Willebrand disease due to increasing platelet count can readily explain the paradox of thrombosis and bleeding in thrombocythemia. Clin Appl Thromb Hemost. 1999;5(3):147-151.

10. Mohri H. Acquired von Willebrand syndrome: features and management. Am J Hematol. 2006;81(8):616-623.

11. Tefferi AA. A long-term retrospective study of young women with essential thrombocythemia. Mayo Clin Proc. 2001;76(1):22-28.

12. Özgönenel B, Rajpurkar M, Lusher JM. How do you treat bleeding disorders with desmopressin? Postgrad Med J. 2007;83(977):159-163. 
Open Access Emergency Medicine

Dovepress

\section{Publish your work in this journal}

Open Access Emergency Medicine is an international, peer-reviewed, open access journal publishing original research, reports, editorials, reviews and commentaries on all aspects of emergency medicine. The manuscript management system is completely online and includes a very quick and fair peer-review system, which is all easy to use.

Submit your manuscript here: http://www.dovepress.com/open-access-emergency-medicine-journal
Visit http://www.dovepress.com/testimonials.php to read real quotes from published authors. 